
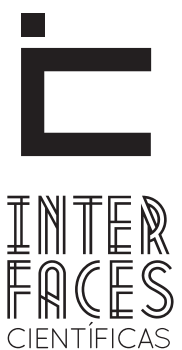

SAÚDE E AMBIENTE

ISSN IMPRESSO 2316-3313

E - ISSN 2316-3798

DOI - 10.17564/2316-3798.2017v5n3p87-96

\title{
PERCEPÇÃO DE MULHERES SOBRE OS FATORES ASSOCIADOS A NÃO REALIZAÇÃO DO EXAME PAPANICOLAU
}

\author{
WOMEN'S PERCEPTION ABOUT THE FACTORS ASSOCIATED WITH NOT PERFORMING THE PAP SMEAR
}

PERCEPCIÓN DE LAS MUJERES SOBRE LOS FACTORES ASOCIADOS A LA NO REALIZACIÓN DE LA PRUEBA DE PAPANICOLAOU

Lilian Christianne Rodrigues Barbosa ${ }^{1}$

Dannyela Andreia Silva ${ }^{3}$

Nadja Romeiro dos Santos 5
Cristiane Maria Alves Silva²

Luana Jeniffer Souza Farias da Costa ${ }^{4}$

\section{RESUMO}

O câncer do colo uterino apresenta elevado índice de mortalidade no Brasil, sendo considerado como um problema de saúde pública mundial. A principal estratégia para prevenção no Brasil é a detecção do câncer in situ, ou de lesões precursoras através do exame de citologia oncótica (Papanicolau). 0 presente artigo teve por objetivo principal conhecer a percepção das usuárias do Sistema Único de Saúde sobre os fatores que dificultam a submissão e periodicidade do exame de citologia oncótica. Trata-se de um estudo descritivo de caráter qualitativo em uma Unidade Básica de Saúde em Maceió/AL, composto por 20 mulheres que nunca haviam se submetido ao exame e as que haviam realizado há mais de três anos. Os resultados apontaram que a desinformação e a dificuldade de marcação do exame, são fatores prevalentes para a não submissão e periodicidade do exame. Nota-se a necessidade de investir na educação em saúde dos profissionais, e ainda, viabilizar estratégias, juntamente com a administração da unidade de saúde, para a melhoria na cobertura do exame pelo Sistema Único de Saúde.

\section{PALAVRAS CHAVE}

Citologia, Neoplasias, Sistema Único de Saúde. 


\section{ABSTRACT}

Cervical cancer has a high mortality rate in Brazil, being considered as a public health problem worldwide. The main strategy for prevention in Brazil is cancer detection in situ, or precursor lesions by examining cytology (Pap smear). This article was aimed at assessing the perception of the users of the Unified Health System on the factors that hinder the periodicity of submission and examination of cytology. This is a descriptive qualitative study in a Basic Health Unit in Maceió / AL, composed of 20 women who had never undergone the examination and who had been held for more than three years. The results indicated that the misinformation and the difficulty of scheduling an examination, are prevalent for non-submission and frequency of the examination factors. Health professionals need of investing in health education, and also to make feasible, together with the administration of the health unit, for an improvement in the coverage of the examination by the Unified Health System.

\section{KEYWORDS}

Cytology. Neoplasms. Unified Health System.

\section{RESUMEN}

El cáncer de cuello uterino tiene una alta tasa de mortalidad en Brasil y es considerado como un problema de salud pública mundial. La principal estrategia para la prevención en Brasil es la detección de lesiones de cáncer in situ o precursoras mediante el examen de la citología (prueba de Papanicolau). Este artículo tuvo como objetivo principal conocer la percepción de los usuarios del Sistema Único de Salud en los factores que dificultan la presentación y la frecuencia del examen de citología. Se trata de un estudio cualitativo descriptivo en una Unidad Básica de Salud en Maceió/AL, compuesto por 20 mujeres que nunca se habían sometido a examen y que habían permanecido durante más de tres años. Los resultados mostraron que la falta de información y la dificultad de la marca examen, son factores predominantes para la no presentación y la frecuencia de los exámenes. Es percibida la necesidad de invertir en educación para profesionales de la salud, y también permite, junto con la administración de las estrategias de las unidades de salud para la mejora de la cobertura de la prueba por el Sistema Nacional de Salud.

\section{PALABRAS CLAVE}

Citología, Neoplasias, Sistema único de Salud. 


\section{INTRODUÇ̄̃̃O}

A Agência Internacional de Pesquisa sobre o Câncer (IARC; 2012), afirmou que o câncer do colo uterino é considerado um problema de saúde pública. No mundo é classificado como a terceira neoplasia maligna mais frequente entre as mulheres e no Brasil a quarta causa de morte de mulheres por câncer (BRASIL, 2016a). Países em desenvolvimento apresentam maiores incidências, correspondendo a 528.000 novos casos ocorridos em 2012 (AKINYEMIJU, 2012).

Configura-se como uma importante causa de óbito nas regiões Norte e Nordeste do Brasil. No ano de 2012, surgiram no Brasil 17.540 novos casos de câncer colo do útero, com uma estimativa de risco de 17 para cada 100.000 mulheres (BRASIL, 2012). O Instituto Nacional de Câncer (INCA) estimou 6.340 novos casos para o ano de 2016. Apesar do investimento de mais de 10 milhões na realização do exame citopatológico por ano e mesmo com os avanços do SUS, o Brasil continua com importante índice de mortalidade por câncer cervical, continuar a reduzir a mortalidade por esta causa, ainda é um desafio a ser vencido (BRASIL, 2015; BRASIL, 2016b).

O câncer de colo uterino é uma neoplasia maligna que acomete a porção inferior do útero denominada cérvix. Doença progressiva e de lenta evolução, que apresenta uma fase pré-invasiva denominada de neoplasia intraepitelial cervical (NIC), esta fase é categorizada em graus I, II, III que vai depender do tecido acometido, sendo o II e III os estágios mais graves (BRASIL, 2011b; SANTOS, 2015).

Os principais fatores de riscos para o desenvolvimento dessa enfermidade são: atividade sexual precoce, múltiplos parceiros, tabagismo e o uso prolongado de pílulas anticoncepcionais (BRASIL, 2016a). As formas de prevenção incluem a prevenção primária e secundária, a primária objetiva a eliminação ou minimização de exposições aos fatores de risco e a secundária é a detecção do câncer in situ ou de lesões precursoras destes (SOUZA, 2008).

A prevenção primária está pautada na diminuição do risco de contágio pelo papilomavírus humano
(HPV), para tanto é necessário o uso de preservativo (camisinha) durante todas as relações sexuais. A infecção persistente pelo vírus HPV especialmente por subtipos HPV-16 e o HPV-18 está associada ao desenvolvimento de lesões intraepiteliais de alto grau e do câncer do colo do útero (WHO, 2010; SANTOS, 2015; BRASIL, 2016a).

A prevenção secundária é feita por meio do exame citopatológico cervical conhecido também como Papanicolaou, considerado como principal estratégia para detecção de lesões precoce e definição do diagnóstico da doença antes do surgimento dos sintomas. A realização periódica permite o diagnóstico precoce e redução da mortalidade por câncer do colo do útero (BRASIL, 2011a, 2011c).

A periodicidade da realização do exame preventivo, estabelecida pelo Ministério da Saúde está de acordo com as recomendações dos principais programas internacionais de controle do câncer que traz: a faixa etária que deve-se ter como prioridade para realização do exame é entre 25 a 60 anos de idade, sendo realizado uma vez por ano e, após dois exames anuais consecutivos com resultados negativos, a cada três anos, torna-se importante resaltar que toda mulher que já teve sua primeira coitarca deve submeter-se ao exame até os 64 anos de idade (BRASIL, 2011b; BRASIL, 2013).

De acordo com a literatura científica os principais motivos para não realização do exame de citologia oncótica são: pouca informação sobre a doença, presença de pudores, tabus, medo, dificuldade na acessibilidade aos serviços de saúde, déficit na qualidade dos exames e condições socioeconômicas e culturais desfavoráveis (FERNANDES et al., 2009; RICO; IRIART, 2013).

Cunha e Vieira-da-Silva (2010), reforçaram como empecilho para realização do exame o agendamento da consulta que provoca dificuldade no acesso à atenção básica em cidades do Nordeste do Brasil, em unidades tradicionais e de saúde da família.

Apesar de várias estratégias de prevenção, promoção e reabilitação do câncer de colo uterino e alta taxa de cura, o índice de mortalidade por esta causa permanece elevado, evidenciando que as mulheres estão descobrindo o câncer de forma tardia, não se subme- 
tendo a prevenção (BRASIL, 2016b). O presente estudo buscou conhecer a percepção de mulheres sobre os fatores que dificultam a submissão e periodicidade do exame de citologia oncótica, uma vez que este funciona como a principal estratégia de prevenção e detecção precoce do câncer cérvico vaginal.

\section{MÉTODOS}

Trata-se de um estudo descritivo com abordagem qualitativa, realizado em uma Unidade Básica de Saúde no município de Maceió-AL. Estudo com característica censitária, onde foram entrevistadas todas as mulheres presentes na unidade nos dias em que ocorreram a coleta de dados. Como critérios de inclusão foram consideradas as mulheres que nunca submeteram ao exame e as que haviam realizado há mais de três anos, com idade entre 25 a 60 anos, participando do estudo 20 mulheres. A justificativa para escolha da faixa etária foi definida a partir das orientações do Ministério da Saúde, que indica este grupo etário como preferencial na prevenção do câncer de colo uterino, devido à incidência de ser maior neste grupo (BRASIL, 2013). Sendo excluídas as mulheres que mantinham a periodicidade regular do exame e as que não aceitaram participar da pesquisa.

0 período de execução da pesquisa foi de novembro de 2012 a abril de 2013. Sendo realizada a coleta de dados por meio de uma entrevista individual, utilizando como instrumento um questionário estruturado, que abordava os seguintes itens: dados pessoais, tempo de realização do exame, conhecimento sobre a finalidade do procedimento e a percepção sobre fatores que influenciaram para a sua não realização.

Os dados foram analisados por meio da técnica de análise do conteúdo, técnica que possibilita o estudo de motivações, atitudes, valores, crenças e tendências, e trabalha com categorização que emerge da fala dos sujeitos (BARDIN, 2011). Esta utiliza uma metodologia de interpretação, onde extrai sentido dos dados de texto e imagem (CRESWELL, 2007).

Dentro dos aspectos éticos, o estudo obteve apro- vação do Comitê de Ética em Pesquisa da Universidade Estadual de Ciências da Saúde de Alagoas (UNCISAL) sob Protocolo n 1813/2012. As questões éticas foram preservadas em decorrência da assinatura do Termo de Consentimento Livre e Esclarecido (TCLE), respeitando as diretrizes e normas regulamentadoras de pesquisas, envolvendo seres humanos de acordo com a Resolução n 466/2012 do Conselho Nacional de Saúde.

\section{RESULTADOS E DISCUSSÃO}

As mulheres da pesquisa apresentaram faixa etária de 25 a 64 anos, a maioria possuía ensino fundamental incompleto e parceiro único, proveniente de união consensual. Ao examinar o conhecimento dos sujeitos da pesquisa sobre a finalidade do exame de Papanicolau, foi possível identificar três categorias como: prevenção, diagnóstico e desinformação. $\mathrm{Na}$ categoria de prevenção foi possível perceber que as mulheres possuíam conhecimento referente ao objetivo do exame:

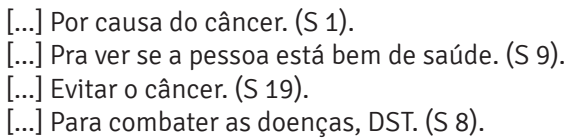

As falas das mulheres entrevistadas demonstraram que estas procuraram realizar o exame por prevenção, apesar de não manter a periodicidade de concretização. 0 exame de citologia oncótica é um método preventivo, pois detecta precocemente as lesões precursoras do câncer e o câncer in situ, sendo considerado a principal estratégia no Brasil (BRASIL, 2011b). Considera-se importante o conhecimento das mulheres sobre a função do exame, pois assim influenciará em sua prática.

Na categoria diagnóstico, as mulheres expressaram que o significado do exame seria detectar doenças.

\footnotetext{
[...] Pra ver se têm câncer (S 3).

[...] Saber se tem alguma doença de ovário, corrimento. (S 4).
} 
[...] Problema de inflamação. (S 5).

[...] Para saber se tem alguma doença. (S 6).

[...] Saber se tem algum problema, se está tudo certo. (S 7).

[...] Para saber se tem doença venérea. (S 10).

[...] Para ver se tem alguma inflamação. (S 13).

[...] Saber como a pessoa está, se tem câncer, alguma inflamação. (S 14).

[...] Para saber se tem alguma inflamação. (S 15).

[...] Saber se tem doença que pega do homem para mulher. (S 16).

[...] Saber se tem câncer, uma inflamação. (S 17).

[...] Detectar alguma coisa no útero. (S 18).

[...] Para detectar doença transmissíveis. (S 20).

Não deixa de ser uma função do exame a detecção de uma doença, visto que sua finalidade é identificar de forma precoce o câncer, porém demonstraram pouco conhecimento quando se trata do real objetivo, onde algumas referem a detecção do câncer e outras para o diagnóstico de outras doenças.

Em um estudo realizado sobre a percepção das mulheres frente ao exame de Papanicolau: da observação ao entendimento, as mulheres estudadas acharam importante a realização do exame pela possibilidade de descobrirem uma possível doença. Esse motivo realça a pouca informação que possuem, não apresentando preocupação com a prevenção do câncer. Essa atitude pode ser provocada pelas informações e campanhas pouco difundidas pelos serviços de saúde e orientação dos profissionais (OLIVEIRA; ALMEIDA, 2009).

A existência da categoria desinformação foi percebida por meio das falas de algumas das mulheres, refletindo na atuação deficiente dos profissionais frente à promoção de informações que sensibilizem as mulheres a procura do serviço para submissão ao exame preventivo. Esta categoria foi expressa pelas mulheres:

\author{
[...] Não sei. (S 2). \\ [...] Não sei, só fiz uma vez e não fiz mais. (S 11). \\ [...] Para vários setores. (S 12).
}

Todas as mulheres entrevistadas estavam presentes em uma unidade básica de saúde para usufruir de algum serviço desta. Isto demonstra que utilizam os serviços ofertados pelo Sistema Único de Saúde (SUS) na Unidade Básica de Saúde (UBS). Percebe-se com isso a ausência de atividades relacionadas à promoção por meio de educação no serviço de saúde, desenvolvida pelos profissionais com a finalidade de investigar e informar sobre o exame preventivo.

Em um estudo realizado sobre o conhecimento cotidiano de mulheres na região do Grande Dirceu em Teresina em relação à prevenção do câncer de colo do útero, mostrou que mesmo o exame sendo disponibilizado de forma periódica, muitas mulheres apresentaram resistência, um dos fatores citado para a não realização foi a desinformação sobre a doença. É preciso que os profissionais trabalhem de forma a desenvolver estratégias que sensibilizem as mulheres, contribuindo na prevenção do câncer, disponibilizando informações sobre o exame e sua periodicidade, a doença e as consequências da não identificação precoce desta (NASCIMENTO, 2010).

É fundamental que os serviços de saúde orientem sobre o que é e qual a importância do exame preventivo, pois sua realização periódica permite que o diagnóstico seja feito cedo e reduza a mortalidade por câncer do colo do útero.

Em relação à opinião das mulheres sobre o motivo que influência a não realização ao exame de citologia oncótica foram incluídas as categorias: acessibilidade, informação, interesse, ausência de sintoma, medo e religião. Na categoria da acessibilidade, as mulheres referiram à dificuldade na marcação do exame no serviço de saúde.
[...] Quando venho marcar não tem ficha. (S 8).
[...] Porque estou tentando marcar, pegar uma ficha né. (S 1).
[...] Toda vez que venho pegar ficha não tem. (S 4). 
[...] Dificuldade para marcar, por morar longe, pegar ficha e não tem. (S 5).

[...] Por conta do tempo e para conseguir uma ficha é uma briga. (S 18).

[...] Dificuldade de marcar. (S 13).

[...] Muito difícil para marcar, pegar uma ficha. (S 17).

[...] Não tem médico, tenta marcar e não tem. (S 10).

[...] Por ser difícil para marcar, passa pelo cora, é difícil para pegar o resultado. (S 11).

Em um estudo sobre a cobertura e fatores associados a não realização do Papanicolau em um município do Sul do Brasil, entre $99 \%$ de mulheres que estavam com a situação em atraso, um dos principais motivos relatados para a não adesão ao exame foi a dificuldade para o agendamento do mesmo (DUAVY et al., 2007).

Segundo Silva, Silveira e Gregório (2012), em estudo realizado sobre motivos alegados para a não realização do exame de Papanicolau, foram apresentados pelas mulheres: pouco acesso nas unidades de saúde para realização do exame, funcionamento do exame no horário de trabalho, falta de material para realização do exame e dificuldade no agendamento.

Uma pesquisa referente a integralidade no cuidado ao câncer do colo do útero e a avaliação do acesso ao serviço de saúde, apontou que a dificuldade de acesso aos serviços de atenção básica e a busca ativa insuficiente são os motivos para a não submissão ao exame. Relatou ainda, que a dificuldade no acesso está relacionada à baixa flexibilidade no agendamento de consultas, medidas estas impostas pelo atendimento que contribuem para dificultar e desmotivar a busca do serviço pelas mulheres, retardando a realização do Papanicolau (SILVA et al., 2014). Percebe-se então uma deficiência na cobertura do exame pelo SUS, dificultando assim a acessibilidade das mulheres a submeterem ao exame preventivo.

A detecção precoce do câncer cérvico-uterino é realizada nas Unidades Básicas de Saúde, nível de baixa complexidade, exigindo menos recursos financeiros disponibilizados pelo SUS, em quanto o câncer in situ e o invasor exigirá atendimento e tratamento em um nível de complexidade intermediário e até de alta complexidade como no caso da histerectomia (BRASIL, 2013; BRASIL, 2016b).

Investir na organização da recepção das UBS e implementar estratégias que visem ampliar e facilitar 0 acesso das mulheres como: permitir 0 atendimento sem agendamento, diversidade no horário e dias de atendimento (incluindo horário noturno e finais de semana) e busca ativa das mulheres (PARADA et al., 2008 apud SILVA et al., 2014). A segunda categoria apresentada foi a informação, demonstrando pouco conhecimento sobre o exame e sua importância.

[...] Por não saber. (S 16).

[...] Não sei dizer, o tempo vai passando. (S 20).

A falta de conhecimento adequado sobre o câncer de colo uterino e do exame preventivo interfere na procura para realização do exame. A desinformação provoca uma despreocupação e consequente falta de interesse pela prevenção, não apenas do câncer, mas também de outras patologias (CHUBACI; MERIGHI; YASUMORI, 2005; RESSEL et al., 2013). Diante disso, confirma-se a necessidade da atuação do profissional em informar sobre o câncer de colo uterino, importância da prevenção e consequências da não submissão e periodização ao exame, assim as mulheres assumiram consigo o compromisso na busca pelo autocuidado.

É necessária a estruturação dos serviços de saúde para que possa ofertar orientação à população a respeito do exame de citologia oncótica, visto que a periodização do exame reduz a mortalidade por câncer do colo do útero na população de risco. Nota-se também a importância de como são transmitidas as informações, necessitando de uma linguagem clara, humanizada, acolhedora por parte dos profissionais, pois a forma como estas são passadas para as mulheres influenciará na sua adesão pela busca do cuidado (RESSEL et al., 2013). 
$\mathrm{Na}$ terceira categoria, trata-se do interesse, onde as mulheres expressaram uma falta de interesse em realizar o exame.

[...] Falta de tempo, dinheiro, dificuldade para esperar para fazer. (S 7).

[...] Falta de descuido, por achar que estava bem. (S 15).

[...] Porque não quis, nunca vou ao ginecologista. (S 2).

O desinteresse em realizar o exame revela o desconhecimento sobre o mesmo, sua finalidade e importância. A falta de informação pode causar despreocupação e como consequência a falta de interesse pela prevenção, não só do câncer de colo uterino, como também de outras doenças ginecológicas (CHUBACl; MERIGHI; YASUMORI, 2005).

A categoria quatro foi classificada de acordo com a ausência de sintomas para a não submissão ao exame, expressos nas falas:

\section{[...] Por não sentir nada. (S 9). \\ [...] Por nunca sentir nada. (S 19). \\ [...] Por nunca sentir nada. (S 6).}

Procurar realizar o exame apenas quando apresenta algum sintoma, revela a falta de conhecimento por parte destas mulheres sobre o câncer e a importância do exame.

As lesões no colo uterino não apresentam sintomas característicos, só apareceram às manifestações clínicas quando ocorre a evolução para o câncer in situ (SILVA et al., 2005). 0 exame tem o objetivo de diagnosticar precocemente o câncer (BRASIL, 2012), este rastreamento em mulheres assintomáticas faz parte de iniciativas do Programa Nacional de Controle do Câncer de Colo uterino no Brasil (BRASIL, 2010). Assim, é necessário que os profissionais ao realizarem a educação em saúde sobre a prevenção expliquem sobre a importância da periodização do exame mesmo sem apresentar sintomas.

A quinta categoria foi em relação ao medo em realizar o exame, expresso na fala: "[...] Por medo, a primeira vez que fiz não gostei” (S 3). 0 medo é con- siderado como um dos tabus mais frequente entre as mulheres para a dificuldade na hora da realização do exame, devido ainda algumas delas demonstrarem receio em sentir dor ou vergonha (SILVA et al., 2008).

O profissional de saúde possui um papel fundamental em relação à prevenção, necessitando desempenhar um papel de educador, trabalhando os fatores que ainda influenciam as mulheres no seu autocuidado. Ainda existem sentimentos de vergonha, preconceito e falta de informação em relação à realização do procedimento. Por meio do esclarecimento prestado às mulheres, ocorrerá uma modificação em sua percepção sobre o Papanicolau, passando a realizar de forma rotineira e frequente em suas vidas (SILVA et al., 2008).

Segundo a fala da sujeita, esta já havia realizado o exame uma vez, momento em que o profissional deveria ceder todas as informações referente a periodização do exame e também ter a sensibilidade de perceber se a mulher apresentou, receio, medo ou até mesmo o desconforto para explicá-la melhor sobre o procedimento e a importância de sua realização.

A categoria seis foi definida pela religião como empecilho para periodização do Papanicolau. Expressada pela fala: "[...] Porque entrei na vida religiosa e não tive mais como continuar" (S 12). Ao participar de uma religião e deixar de realizar o exame de prevenção revela a falta de informação desta, sobre a doença e a importância de se manter a regularização do exame.

Em sociedades diferentes, o corpo feminino é regulado por meio de normas, sejam elas baseadas em crenças, mágicas, religião ou Medicina (DUAVY et al., 2007). Cabe ao profissional saber lidar com os diferentes aspectos apresentados por cada mulher, enfatizando e deixando claro para estas a importância de manter o cuidado da saúde.

Em um estudo sobre os fatores que influenciam a realização do exame preventivo do câncer cérvico-uterino em Porto Velho, revelou a existência de preferência de participantes pela submissão ao exame preventivo nas igrejas, por ser um espaço físico e social de acolhimento. Percebe-se a necessidade de implementação de Políticas intersetoriais desenvolvidas para aumentar as taxas de cobertura do exame nestas instituições (LUCENA et al., 2011). 


\section{CONCLUSÃO}

Diante dos resultados apresentados foi possível identificar a desinformação e a dificuldade de marcação do exame como fatores prevalentes. A falta de atuação na educação em saúde e a busca ativa das mulheres na comunidade foram pontos dignos de ajuste nas estratégias de atuação, por parte dos profissionais de saúde. Percebe-se a necessidade de uma atuação mais ativa do profissional em explicar sobre o câncer de colo do útero, o exame preventivo, a importância de sua realização e as consequências da não periodização do exame. Atuando não mais de forma mecanicista como o modelo biomédico que só busca a cura, mas operando de forma mais humana e acolhedora, para que possam adquirir a confiança das mulheres e sensibilizarem a tomar uma atitude de cuidar de sua saúde.

Em relação à dificuldade de marcação nota-se a necessidade de melhoria na administração do agendamento desse exame, assim como intensificar a qualidade da cobertura deste pelo SUS nas UBS.

Sabe-se que o custo para o tratamento como a radioterapia, quimioterapia e/ou cirurgia são bem mais elevados do que quando relacionado à prevenção, então investir na prevenção irá não só reduzir os gastos como também o índice de morbimortalidade por câncer de colo uterino que ainda é alto.

\section{REFERÊNCIAS}

AKINYEMIJU, T.F. Socio-economic and health access determinants of breast and cervical cancer screening in low-income countries: analysis of the world health survey. PLoS One, San Francisco, v.7, n.11, e48834, 2012.

BARDIN, L. Análise de conteúdo. Tradução Luis Antero Reto, 70.ed. São Paulo: Augusto Pinheiro, 2011.

BRASIL. Ministério da Saúde. Instituto Nacional de
Câncer. Plano de ação para redução da incidência e mortalidade por câncer do colo do útero: sumário executivo. Rio de Janeiro: INCA, 2010.

BRASIL. Ministério da Saúde. Biblioteca Virtual em Saúde. Exame preventivo do câncer de colo uterino (Papanicolau). Brasília: BVSMS, 2011a.

BRASIL. Ministério da Saúde. Instituto Nacional de Câncer. Colo do útero: detecção precoce. Rio de Janeiro: INCA, 2011b.

BRASIL. Ministério da Saúde. Instituto Nacional de Câncer. Coordenação Geral de Ações Estratégicas.

Diretrizes brasileiras para o rastreamento do câncer do colo do útero. Divisão de Apoio à Rede de Atenção Oncológica. Rio de Janeiro: INCA, 2011c.

BRASIL. Ministério da Saúde. Instituto Nacional de Câncer. Estimativas 2012: incidência de câncer no Brasil. Rio de Janeiro: INCA, 2012.

BRASIL. Ministério da Saúde. Secretaria de Atenção à Saúde. Controle dos cânceres do colo do útero e da mama. 2.ed. Brasília: Departamento de Atenção Básica, 2013.

BRASIL. Ministério da Saúde. Instituto Nacional de Câncer José Alencar Gomes da Silva. Estimativa 2016: incidência do câncer no Brasil. Rio de Janeiro: INCA, 2015.

BRASIL. Ministério da Saúde. Instituto Nacional de Câncer José Alencar Gomes da Silva. Câncer do colo do útero. Rio de Janeiro: INCA, 2016a.

BRASIL. Ministério da Saúde. Instituto Nacional de Câncer José Alencar Gomes da Silva. Diretrizes brasileiras para o rastreamento do câncer do colo do útero. Divisão de Detecção Precoce e Apoio à Organização de Rede. 2.ed. rev. atual. Rio de Janeiro: INCA, 2016b. 
CHUBACI, Y. S.; MERIGHI, M. A. B.; YASUMORI, Y. A mulher japonesa vivenciando o câncer cérvicouterino: um estudo de caso com abordagem da fenomenologia social. Rev. Esc. Enferm. USP, São Paulo, v.39, n.2, p.189-94, 2005.

CRESWELL, J. W. Projeto de pesquisa: métodos qualitativo, quantitativo e misto. Tradução L.O. Rocha. 2.ed. Porto Alegre: Artmed, 2007.

CUNHA, A.B.O.; VIEIRA S.L.M. Acessibilidade aos serviços de saúde em um município do Estado da Bahia, Brasil, em gestão plena do sistema. Cad.

Saúde Pública, Rio de Janeiro, v.26, n.4, p.725-737, 2010.

DUAVY, L.M. et al. A percepção da mulher sobre o exame preventivo do câncer cérvico-uterino: estudo de caso. Ciên. Saúde Colet., Rio de Janeiro, v.12, n.3, p.733-742, 2007.

FERNANDES, J.V. et al. Conhecimentos, atitudes e prática do exame de Papanicolaou por mulheres no Nordeste do Brasil. Rev.Saúde Públ., São Paulo, v.43, n.5, p.851-858, 2009.

IARC - International Agency for Research on Cancer. World Health Organization. GLOBOCAN 2012.

Estimated cancer incidence, and mortality and prevalence worldwide in 2012: cancer fact sheets. Lyon: IARC, 2013.

LUCENA, L.T. et al. Fatores que influenciam a realização do exame preventivo do câncer cérvico uterino em Porto Velho, Estado de Rondônia, Brasil. Rev Pan-Amaz Saúde, Ananindeua, v.2, n.2, p.45-50, 2011.

NASCIMENTO, C.L. Representações sociais da prevenção do câncer cérvico-uterino elaboradas por mulheres. 2010. 95f. Dissertação (Mestrado) Universidade Federal do Piauí, Teresina, 2010.

OLIVEIRA, S.L.; ALMEIDA, A.C.H. A percepção das mulheres frente ao exame de papanicolaou: da observação ao entendimento. Cogitare Enferm. Curitiba, v.14, n.3, p.518-526, 2009.

RESSEL, L.B. et al. Exame preventivo do câncer de colo uterino: a percepção das mulheres. Av. Enferm., Bogotá, v.31, n.2, p.65-73, 2013.

RICO, A.M.; IRIART, J.A.B. “Tem mulher, tem preventivo": sentidos das práticas preventivas do câncer do colo do útero entre mulheres de Salvador, Bahia, Brasil. Cad. Saúde Públ., Rio de Janeiro, v.29, n.9, p.1763-1773, 2013.

SILVA, K.B. et al. Integralidade no cuidado ao câncer do colo do útero: avaliação do acesso. Rev. Saúde Públ., São Paulo, v.48, n.2, p.240-248, 2014.

SILVA, S.R.; SILVEIRA, C.F.; GREGÓRIO, C.C.M. Motivos alegados para a não realização do exame de Papanicolaou, segundo mulheres em tratamento quimioterápico contra o câncer do colo uterino. Rev. Min. Enferm., Belo Horizonte, v.16, n.4, p.579-587, 2012.

SILVA S.E.D. et al. Representações sociais de mulheres amazônidas sobre o exame papanicolau: implicações para a saúde da mulher. Esc Anna Nery Rev Enferm., Rio de Janeiro. v.12, n.4, p.685-692, 2008.

WHO - World Health Organization. Information Centre on Human Papilloma Virus (HPV) and Cervical Cancer. Human papillomavirus and related cancers in Brazil. Summary Report. 2010. 
1 Universidade Estadual de Ciências da Saúde de Alagoas. Email: Lilian_ cbarbosa@hotmail.com

2 Universidade Estadual de Ciências da Saúde de Alagoas. Email: cmamartins@gmail.com

Recebido em: 10 de Outubro de 2016 Avaliado em: 13 de Outubro de 2016 Aceito em: 28 de Outubro de 2016
3 Universidade Estadual de Ciências da Saúde de Alagoas. Email: danny_ enfon@hotmail.com

4 Universidade Estadual de Ciências da Saúde de Alagoas. Email: luasouza@hotmail.com

5 Secretaria Municipal de Saúde de Maceió - SMS. Email: nadjaromeiro@ gmail.com 